\title{
Re-shaping Healthcare Systems
}

\author{
Bernd Blobel $^{1,2,3}$ and Alexander Berler ${ }^{4,5}$ \\ ${ }^{1}$ Medical Faculty, University of Regensburg, Germany \\ ${ }^{2}$ eHealth Competence Center Bavaria, Deggendorf Institute of Technology, Germany \\ ${ }^{3}$ First Medical Faculty, Charles University Prague, Czech Republic \\ ${ }^{4} \mathrm{HL7}$ Hellas, Athens, Greece \\ ${ }^{5}$ Gnomon Informatics SA, Thessaloniki, Greece
}

\section{Correspondence to:}

Prof. Dr. habil. Bernd Blobel, FACMI, FACHI, FHL7, FEFMI, MIAHSI

Medical Faculty, University of Regensburg, Germany

E-mail: bernd.blobel@klinik.uni-regensburg.de
EJBI 2017; 13(1):1-7

published: October 10, 2017
This Special Issue of the European Journal for Biomedical Informatics is dedicated to the International HL7 Interoperability Conference (IHIC 2017) "Re-shaping healthcare systems", 22-24 October 2017 in Athens, Greece (http://www.ihic2017.eu). It contains papers selected by an independent peer review process, strictly performed by experts from countries different from the authors' country of residence.

IHIC 2017 is the $17^{\text {th }}$ event of the International HL7 Interoperability Conference series, which has been inaugurated in 2000 by the Board of HL7 Germany and its unforgettable Chair and interoperability pioneer Joachim W. Dudeck. The first event in Dresden, Germany, was entitled "Advanced Healthcare Information Standards". While the first conferences have been characterized by focusing on CDA, over the time, the scope of the conferences has been extended towards all aspects of health information interoperability. The concept of interoperability has dramatically changed from standardized electronic data interchange (EDI) based on data representation at application level, the $7^{\text {th }}$ level of the ISO Open Systems Interconnection stack, having been the name giver for the Health Level 7 standards framework. Meanwhile, the semantics of shared data as well as service level interoperability, but also domain-specific issues and even social aspects are considered, bringing terminologies and ontologies, but also implementation and conformance challenges on board. The relations to IHE and the FHIR success are especially highlighted at IHIC 2017. So it is just consequent to address also in 2017 both technological and non-technological issues of interoperability.

IHIC 2017 has been structured into four sections: a) Paradigm Changes in Healthcare and Resulting
Interoperability Challenges, b) Infrastructure and Services for Healthcare Transformation, c) CDA-related Contributions, and d) Other issues. The thematic sections have been introduced by related Keynotes. The papers published in this EJBI Special Issue address the different aspects of the interoperability challenge from a theoretical and methodological perspective, usability requirements, professional groups' preferences, process design, semantical ambiguity, and implementation details..

In his Keynote "A New World for Better Health", Edward Hammond, Duke University (USA), addresses the ongoing healthcare systems' paradigm changes towards accountable care, a patient-centered focus, extensive data sharing, multiple data types, intensive collaboration, open source environments, and mobile technologies to better care an aging society. Thereby, he also highlights related evolving standards such as Fast Healthcare Interoperability Resources (FHIR). Bernd Blobel from the University of Regensburg (Germany), who addressed the aforementioned paradigm changes in health systems over many years already, discusses in his this year's Keynote "Standardization for Mastering Healthcare Transformation - Challenges and Solutions" the interoperability between existing and emerging standards and specifications. Based on a system-theoretical, architecturecentric, ontology-based approach to interoperability, he presents a meanwhile at ISO and CEN standardized Interoperability Reference Architecture Model. He demonstrates the feasibility of that model for cross-domain, i.e. multi-disciplinary, interoperability for integrating so different standards like ISO 13606 "EHR communication", ISO 22600 "Privilege management and access control" and ISO 21298 "Structural and functional roles". 
In the first section on Paradigm Changes in Healthcare and Resulting Interoperability Challenges, Fabrizio Pecoraro and colleagues from the National Research Council, Rome (Italy) describe the Health at Home project to provide health and social care at home, using existing standards for enabling a cross-domain integration and continuity of care perspective over distance. The ContSys standard is used to harmonize the presentation of the involved concepts, and FHIR to implement the solution for information exchange. As security and privacy services including the resulting among the stakeholders as well between them and the patient are crucial for successful health services, Alexander Mense and Bernd Blobel discuss standards and component to support the implementation of the European General Data Protection Regulation (GDPR).

In the second section on Infrastructure and Services for Healthcare Transformation, Junquiao Chen et al. report about a U.S. population health partnership, exchanging and reconciling clinical decision support outcome among a cross-functional, distributed care team. CDS output is automatically harmonized between an EHR system and a population health services platform. That way, care gaps could be identified and overcome, so improving quality of care. Roberta Gazzarata et al. present a regional HIE solution for the Veneto region in North Italy to share clinical data. Thereby, a health terminology service based on CTS2 - a specification jointly developed by HL7 and the Object Management Group (OMG) - for correctly managing semantics is deployed to exchange laboratory reports encoded in LOINC and implemented in CDA documents. Dimitrios Katehakis et al. demonstrate the use of a Personal Health Record (PHR) system for managing chronic diseases, home care and wellbeing. For enabling interoperability between all actors involved, the HL7 PHR Functional Model and Meaningful Use criteria are applied. Finally, Giorgia Gazzarata et al. present a smart solution for access control in distributed EHR systems. That way, trustworthy communication and cooperation in a regional cancer care network will be enabled. Thereby, different access control models and different maturity levels of security and privacy services can be harmonized and consistently managed. The solution is based on a system-theoretical, architecture-centric, ontology -driven solution for policy mapping is deployed.

In the third section on CDA-related Contributions, Elisabeth Pantazoglou et al. from the Niederrhein University of Applied Sciences, Krefeld (Germany) describe a new project for communicating genomic-related medical data. Based on a process analysis, the concepts deployed will be represented by semantic annotation in an international terminology and thereafter implemented using HL7 CDA. Thus, the presented GENeALYSE project will support documentation and communication between diagnostics, medical therapy and research in the field of genome analysis for oncological diseases. Abderrazek Boufahja and Eric Poiseau from IHE Europe, Paris (France) investigate richness of CDA R2 documents and validate the reliability of related test and certification programs. Based on the investigation of existing methods and tools, the authors defined relevant test data, analyzed template inheritance and template containment to derive matrixes for template richness template containment. From that, a richness score of CDA R2 documents as the number of present CDA templates and components related to the number of possible templates and elements is calculated. The results have been demonstrated by assessing the richness of existing CDA implementation guides and approved CDA documents. Fabrizio Pecoraro et al. provide a solution for extracting data from CDA documents, transforming them properly and loading them into a Data Warehouse for secondary use. That way, decision-making for patient safety, healthcare quality assessment, clinical and translational research including clinical trials, comparative analysis of therapy pathways and best practices application can be supported. For this purpose, CDA schemas have to be transformed into a data warehouse dimensional model by mapping the CDA schema primitives with concepts of the dimensional model.

In the final section "Other issues", Robert Snelick from the National Institute of Standards and Technology (NIST), (US), presents a platform for developing specifications, test plans and testing tools to improve conformance and interoperability of solutions. In that context, a set of tools has been presented to define and to constrain HL7 v2 specifications including the development of test plans and test tool building blocks. The offered platform allows the automatic creation of conformance testing tools from those building blocks, that way enabling an end-to-end process improvement.

Additionally to the papers presented here, practice reports and implementation experiences have been shared at the conference. IHIC 2017 was completed by two Tutorial Days covering topics such as CDA, FHIR, but also security and privacy issues, SNOMED-CT and its relations to the TermInfo project as well as IHE in practice.

The Editors wish all interested parties an enjoyable reading.

The Guest-Editors are indebted to thank all authors and reviewers for their excellent work. Finally, they thank HL7 International for sponsoring the event, HL7 International HL7 and Germany for financing the Joachim W. Dudeck Award as well as HL7 Germany and HL7 Hellas for monetarily supporting the Tutorials. 